\title{
Molecular basis of genetic diversity among cytoplasms of Triticum and Aegilops species. IV. CtDNA variation in Ae. triuncialis*
}

\author{
Koji Murai and Koichiro Tsunewaki
}

Laboratory of Genetics, Faculty of Agriculture, Kyoto University, Sakyo-ku, Kyoto 606, Japan.

Restriction endonuclease analysis of chloroplast DNA of Ae. triuncialis was carried out. Thirteen accessions had the type 2 a chloroplast genome derived from Ae. caudata, eight possessed type 3 chloroplast genome of Ae. umbellulata, and the remaining five contained a new chloroplast genome (named type $2 b$ ) differing from the former two, by a $0.3 \mathrm{kbp}$ insertion and four base substitutions, respectively. The accessions with type 2a and type 3 chloroplast genomes distribute in wide areas, and in both of its subspecies, eu-triuncialis and orientalis, whereas those having the type $2 \mathrm{~b}$ chloroplast genome occur only locally in Azerbaijan, Transcaucasus. From these results, the following two conclusions are drawn; (a) Ae. triuncialis originated from the reciprocal crosses between Ae. caudata and Ae. umbellulata, and (b) the type $2 b$ chloroplast genome was arisen from type 2 a chloroplast genome by a $0 \cdot 3 \mathrm{kbp}$ insertion.

\section{INTRODUCTION}

Aegilops triuncialis, a tetraploid species $(2 \mathrm{n}=28$, genome constitution $\mathrm{C}^{\mathrm{u}} \mathrm{C}^{\mathrm{u}} \mathrm{CC}$ ) is known to have originated as an amphidiploid between two diploids, Ae. caudata $(2 \mathrm{n}=14, \mathrm{CC})$ and Ae. umbellulata $\left(2 \mathrm{n}=14, \mathrm{C}^{\mathrm{u}} \mathrm{C}^{\mathrm{u}}\right)$ (Kihara, 1940; Kihara and Kondo, 1943). This species is adapted to a wide range of environments, and is more widely distributed than the two parental species, i.e., from the western Mediterranean to Central Asia (Croston and Williams, 1981). Following Eig (1929), this species is divided into two subspecies, eu-triuncialis and orientalis. Ssp. eu-triuncialis includes two varieties, typica and constantinopolitana, whereas ssp. orientalis consists of three varieties, assyriaca, persica and anathera.

By comparing the morphological and physiological characters of alloplasmic wheat lines, Mukai et al. (1978) found that two accessions of Ae. triuncialis ssp. eu-triuncialis have genetically different cytoplasms. One of them has the almost identical cytoplasm to that of Ae. umbellulata and the other to that of Ae. caudata. Restriction endonuclease analysis of the ctDNA supported these results (Ogihara and Tsunewaki, 1982, 1983).

\footnotetext{
* Contribution No. 481 from the Laboratory of Genetics, Faculty of Agriculture, Kyoto University, Japan. The work was partly supported by a Grant-in-Aid (No. 60400005) from the Ministry of Education, Science and Culture, Japan.
}

Together these observations indicate a possible diphyletic origin of Ae. triuncialis from the reciprocal crosses between Ae. caudata and Ae. umbellulata (Ogihara and Tsunewaki, 1982).

The present investigation aimed to clarify (a) the extent of ctDNA variation within Ae. triuncialis, (b) the geographical distribution of the accessions having different chloroplast genomes, and (c) the distribution of each chloroplast genome type among different taxa.

\section{MATERIALS AND METHODS}

The accessions used and their taxonomy, collection site and source are shown in table 1 . These were provided by M. Tanaka and S. Sakamoto, Plant Germplasm Institute, Kyoto University; Y. Mukai, Osaka Kyoiku University; and the late Y. Nakai, Laboratory of Genetics, Kyoto University. As a control, two alloplasmic common wheats having the cytoplasm of Ae. caudata or Ae. umbellulata were used.

Intact chloroplasts were isolated from the homogenate of mature or seedling leaves and purified by the 10,40 and 75 per cent Percoll or the 15,30 and 60 per cent sucrose discontinuous gradient centrifugation. The final pellet was resuspended in TE buffer ( $50 \mathrm{mM}$ Tris, $20 \mathrm{mM}$ EDTA, $p \mathrm{H} 8.0$ ) and lysed in 0.2 per cent sodium lauryl 
Table 1 The origin of 26 Ae. triuncialis accessions used in the present investigation and their chloroplast genome types

\begin{tabular}{|c|c|c|c|c|}
\hline $\begin{array}{l}\text { Accession } \\
\text { No. }\end{array}$ & Ssp. and var.* & Collection site & Sourcet & $\begin{array}{l}\text { Chloroplast } \\
\text { genome } \\
\text { type } \div\end{array}$ \\
\hline 02 & orie. assy. & Afghanistan; 20-35 km W of Maimana & KUSE 2517 & 3 \\
\hline 03 & orie. pers. & Afghanistan; $75 \mathrm{~km} \mathrm{~W}$ of Maimana & KUSE 2521 & $2 \mathrm{a}$ \\
\hline 04 & eu-t. cons. & Azerbaijan; $34 \mathrm{~km} \mathrm{~N}$ of Baku & BEC 2924 & $2 b$ \\
\hline 07 & eu-t. cons. & Azerbaijan; $34 \mathrm{~km} \mathrm{~N}$ of Baku & BEC 2927 & $2 b$ \\
\hline 08 & eu-t. cons. & Azerbaijan; $34 \mathrm{~km} \mathrm{~N}$ of Baku & BEC 2928 & $2 b$ \\
\hline 09 & eu-t. typ. & Bulgaria; Albena-1 & M356-1 & 3 \\
\hline 10 & eu-t. typ. & Bulgaria; Albena-2 & M356-2 & 3 \\
\hline 11 & eu-t. typ. & Bulgaria; Albena-3 & M356-3 & 3 \\
\hline 12 & eu-t. typ. & Bulgaria; Baltik-1 & M356-4 & 3 \\
\hline 17 & eu-t. cons. & Greece & $\mathrm{N} 7050$ & $2 \mathrm{a}$ \\
\hline 18 & eu-t. typ. & Iran; $2 \mathrm{~km} \mathrm{~N}$ of Karaj & KUSE 2548 & 3 \\
\hline 19 & orie. assy. & Iraq; $16.4 \mathrm{~km} \mathrm{NW}$ from Almigdadiya & BEM 4951 & $2 \mathrm{a}$ \\
\hline 20 & eu-t. typ. & Rumania & $\mathrm{N} 7045$ & $2 a$ \\
\hline 21 & eu-t. typ. & Rumania & N7046 & $2 \mathrm{a}$ \\
\hline 22 & eu-t. typ. & Spain; Carboneras & 702 & $2 a$ \\
\hline 23 & eu-t. typ. & Spain; Canamares & 710 & $2 a$ \\
\hline 24 & eu-t. typ. & Spain; near Almanza & 734 & $2 \mathrm{a}$ \\
\hline 25 & eu-t. typ. & Tu:key; $24 \mathrm{~km}$ E of Mecitozii & BMUK 6853 & $2 \mathrm{a}$ \\
\hline 26 & eu-t. typ. & Turkey; $27 \mathrm{~km} \mathrm{NW}$ of Kirikkale & BMUK 6864 & $2 a$ \\
\hline
\end{tabular}

${ }^{*}$ Full name: eu-t. $=$ ssp. eu-triuncialis, orie. $=$ ssp. orientalis, typ. $=$ var. typica, cons. $=$ var. constantinopolitana, assy. $=$ var. assyriaca, pers. = var. persica.

$\dagger$ KUSE: Kyoto University Scientific Expedition to the Karakoram and Hindukush, 1955 (Kihara et al., 1965). BMUK: Botanical Mission of the Kyoto University to the Eastern Mediterranean, 1959 (Yamashita and Tanaka, 1961). BEC: Botanical Expedition of Kyoto University to Caucasus, 1966. BEM: Botanical Expedition of Kyoto University to Mesopotamia, 1970.

$\$$ Chloroplast genome type $2 \mathrm{a}$ and 3 are identical to that of Ae. caudata and Ae. umbellulata, respectively.

sarcosinate solution, containing $200 \mu \mathrm{g} / \mathrm{ml}$ protenase K. CtDNA was prepared according to Kolodner and Tewari (1975).

The ctDNA was digested with four restriction endonucleases, BamHI, HindIII, SmaI and XhoI, each of which recognises a specific six-base-pair sequence. These endonucleases were chosen because it had been established previously that they could distinguish the ctDNA of Ae. caudata from that of Ae. umbellulata (Ogihara and Tsunewaki, 1982, 1983). The methods of electrophoresing the digested ctDNA and analysing the restriction fragment pattern are the same as previously described (Ogihara and Tsunewaki, 1982).

\section{RESULTS AND DISCUSSION}

\section{(a) CtDNA variation in Ae. triuncialis}

As shown in fig. 1, the BamHI-, HindIII-, SmaIand Xhol-digests of ctDNA from 26 Ae. triuncialis accessions produced three, two, three and three different restriction fragment patterns, iespectively. These patterns are schematically drawn in fig. 2. These results demonstrate the presence of ctDNA variation in this species.

Ogihara and Tsunewaki $(1982,1983)$ recognised 11 major types plus five subtypes among the chloroplast genomes of 33 Triticum and Aegilops species (43 accessions in total), from the results of restriction endonuclease analysis of their ctDNA with eight endonucleases, including $B a m \mathrm{HI}$, HindIII, SmaI and XhoI. Comparing the present results with theirs, 13 and eight accessions of $A e$. triuncialis have type 2 and 3 chloroplast genomes of Ogihara and Tsunewaki, respectively, and the remaining five accessions are of a new type. The present and their results show that type 2 chloroplast genome is in Ae. caudata and type 3 in $A e$. umbeilulata, which are the two parental species of Ae. triuncialis. The new type chloroplast genome is unexpected given the known origin of this species. 


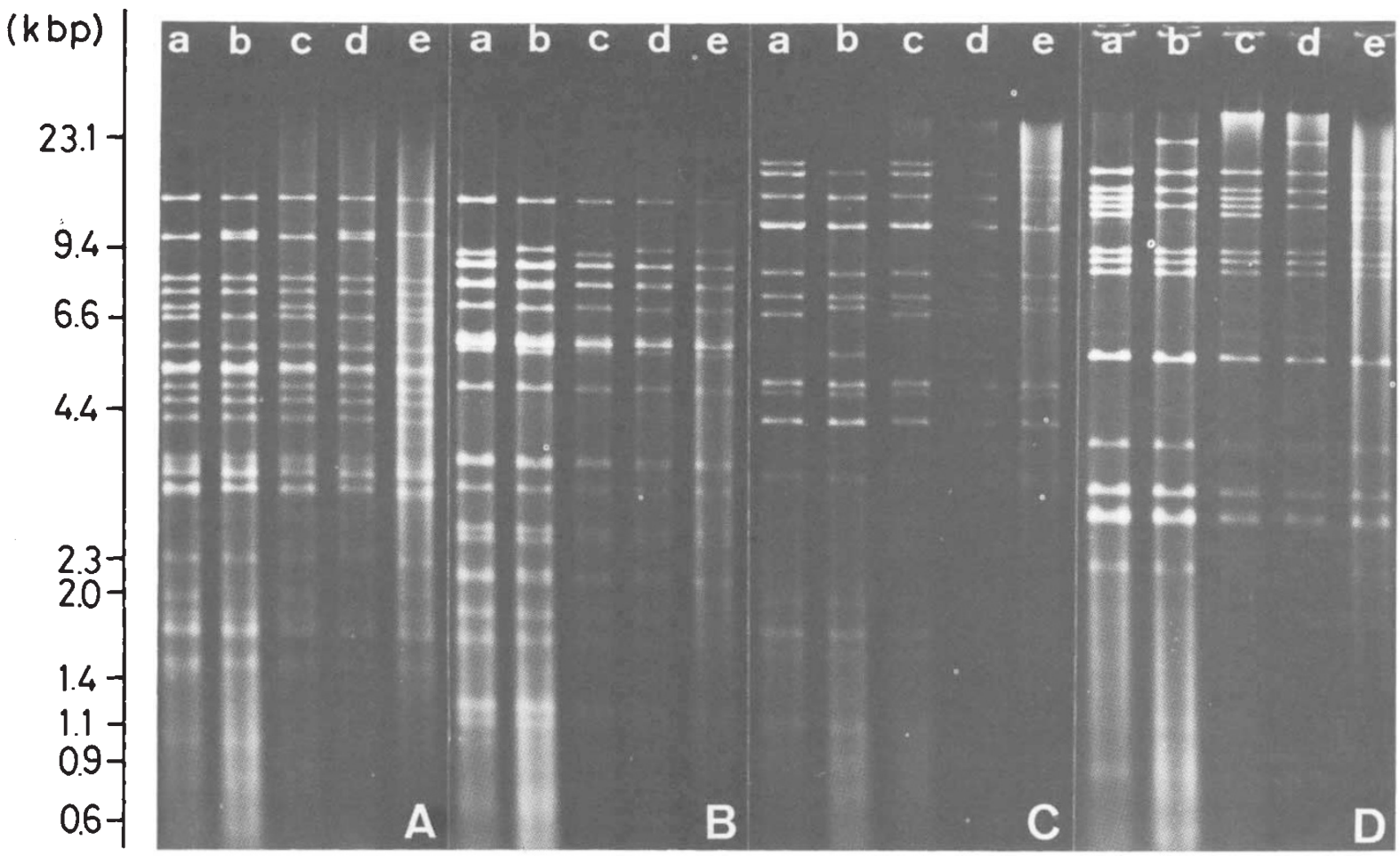

Figure 1 Restriction fragment patterns of ctDNA from three chloroplast genome types of Ae. triuncialis digested by (A) Bam HI, (B) HindIII, (C) SmaI and (D) Xhol. Lane a and b: Alloplasmic common wheat having cytoplasm of Ae. caudata and Ae. umbellulata, respectively. Lane c, d and e: Accession no. 01, 02 and 04 of Ae. triuncialis, representing chloroplast genome type $2 \mathrm{a}, 3$ and $2 \mathrm{~b}$, respectively.

(b) Physical maps of the three chloroplast genomes found in Ae. triuncialis

By reference to the physical map of common wheat ctDNA given by Bowman et al. (1981), Ogihara (pers. comm.) has constructed the physical maps of 16 chloroplast genomes identified in Triticum and Aegilops, and has located the restriction sites of 11 endonucleases together with deletion/insertion sites.

By comparing the present results given in figs 1 and 2 to Ogihara's results, the physical map of the new chloroplast genome was constructed. This is shown in fig. 3 , in which differences of type 2 (=Ae. caudata) and type $3(=$ Ae umbellulata) chloroplast genomes from the new one are also indicated. The new chloroplast genome shows only one difference, namely, a $0.3 \mathrm{kbp}$ insertion from the Ae. caudata chloroplast genome, whereas it shows four restriction site differences from that of Ae. umbellulata, i.e., a gain of two Bam $\mathrm{HI}$ and one XhoI site and the loss of a SmaI site. Apparently, this chloroplast genome is more closely related to the Ae. caudata than to the Ae. umbellulata chloro- plast genome. Thus, the chloroplast genome type newly found in five accessions of Ae. triuncialis is considered to be a subtype of chloroplast genome type 2 , and is named type $2 \mathrm{~b}$. Accordingly, chloroplast genome of Ae. caudata will be renamed type $2 \mathrm{a}$.

\section{(c) Distribution of the three chloroplast genomes in Ae. triuncialis}

Distribution in different taxa: Chloroplast genome types of all Ae. triuncialis accessions are indicated in the last column of table 1 . Table 2 summarises the distribution of the three chloroplast genome types in different taxa of Ae. triuncialis. Both type $2 \mathrm{a}$ and 3 chloroplast genomes are found in the two subspecies, eu-triuncialis and orientalis. Type $2 \mathrm{~b}$ chloroplast genome is found only in ssp. eu-triuncialis var. constantinopolitana.

Geographical distribution: fig. 4 shows the collection sites of Ae. triuncialis accessions having different chloroplast genomes, together with the distribution areas of this species and its two 


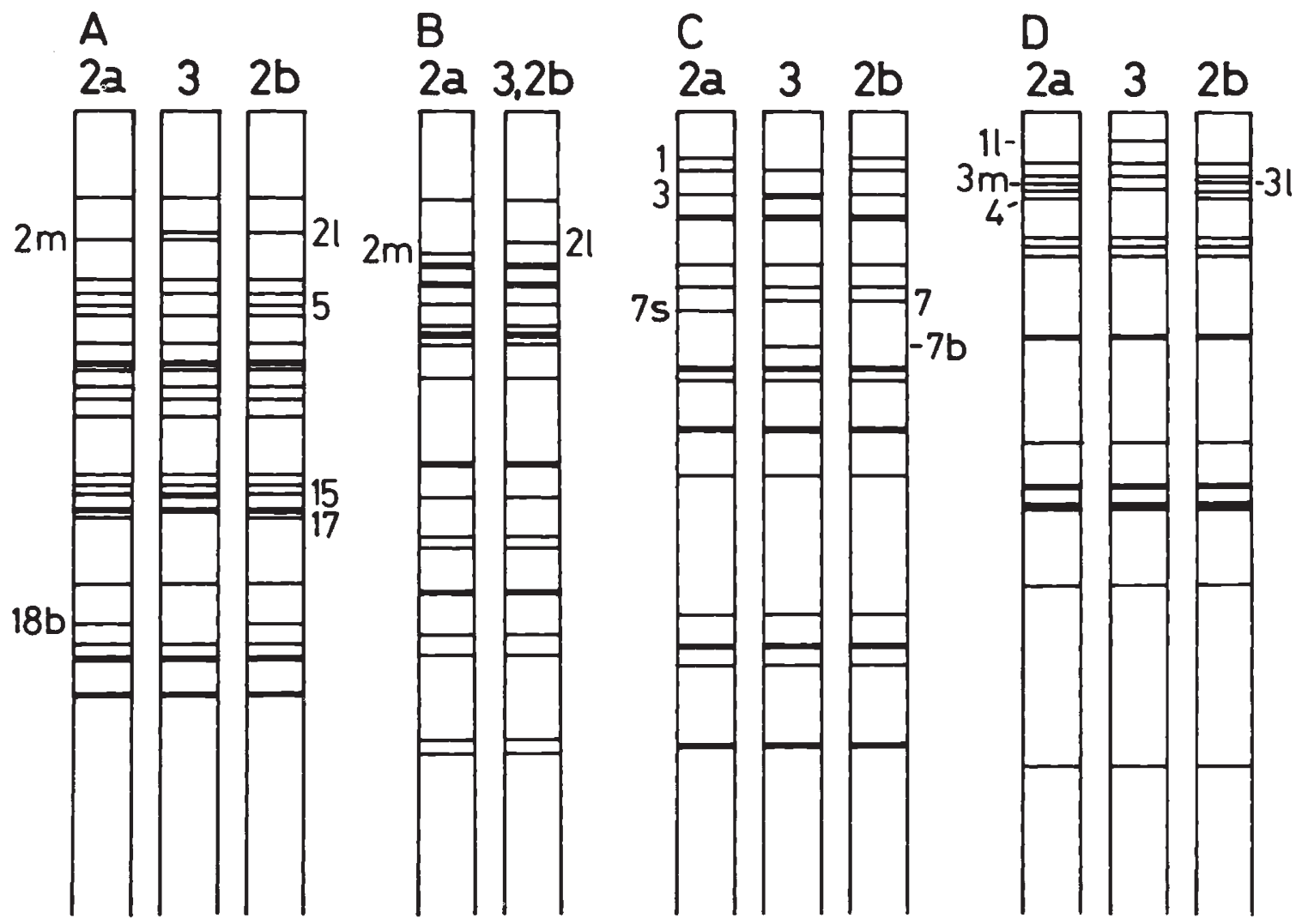

Figure 2 Schematic representation of (A) BamHI, (B) HindIII, (C) SmaI and (D) Xhol restriction patterns of ctDNA from three chloroplast genome types, $2 \mathrm{a}, 2 \mathrm{~b}$ and 3 . Variable fragments are indicated in the respective lanes.



Figure 3 Physical map of the type $2 b$ ctDNA, differences of which from those of type $2 a$ and 3 ctDNA are also indicated. parents. Type 2a and type 3 chloroplast genomecarriers occur widely in the distribution area of Ae. triuncialis. Although type $2 \mathrm{~b}$ chloroplast genome-carrier was found only in Azerbaijan, Transcaucasus, the possibility that it is present in other areas cannot be excluded because of the small sample sizes we studied.

Table 2 Numbers of Ae. triuncialis accessions having different chloroplast genomes

\begin{tabular}{lrrrr}
\hline & \multicolumn{4}{c}{ Chloroplast genome } \\
\cline { 3 - 5 } $\begin{array}{l}\text { Subspecies } \\
\text { and variety }\end{array}$ & $2 \mathrm{a}$ & $2 \mathrm{~b}$ & 3 & Total \\
\cline { 3 - 5 } $\begin{array}{l}\text { Ssp. eu-triuncialis } \\
\quad \text { var. typica }\end{array}$ & 9 & 0 & 7 & 16 \\
$\quad$ var. constantinopolitana & 2 & 5 & 0 & 7 \\
$\quad$ subtotal & 11 & 5 & 7 & 23 \\
Ssp. orientalis & 1 & 0 & 1 & 2 \\
$\quad$ var. assyriaca & 1 & 0 & 0 & 1 \\
$\quad$ var. persica & 2 & 0 & 1 & 3 \\
$\quad$ subtotal & 13 & 5 & 8 & 26 \\
Total & & & & \\
\hline
\end{tabular}




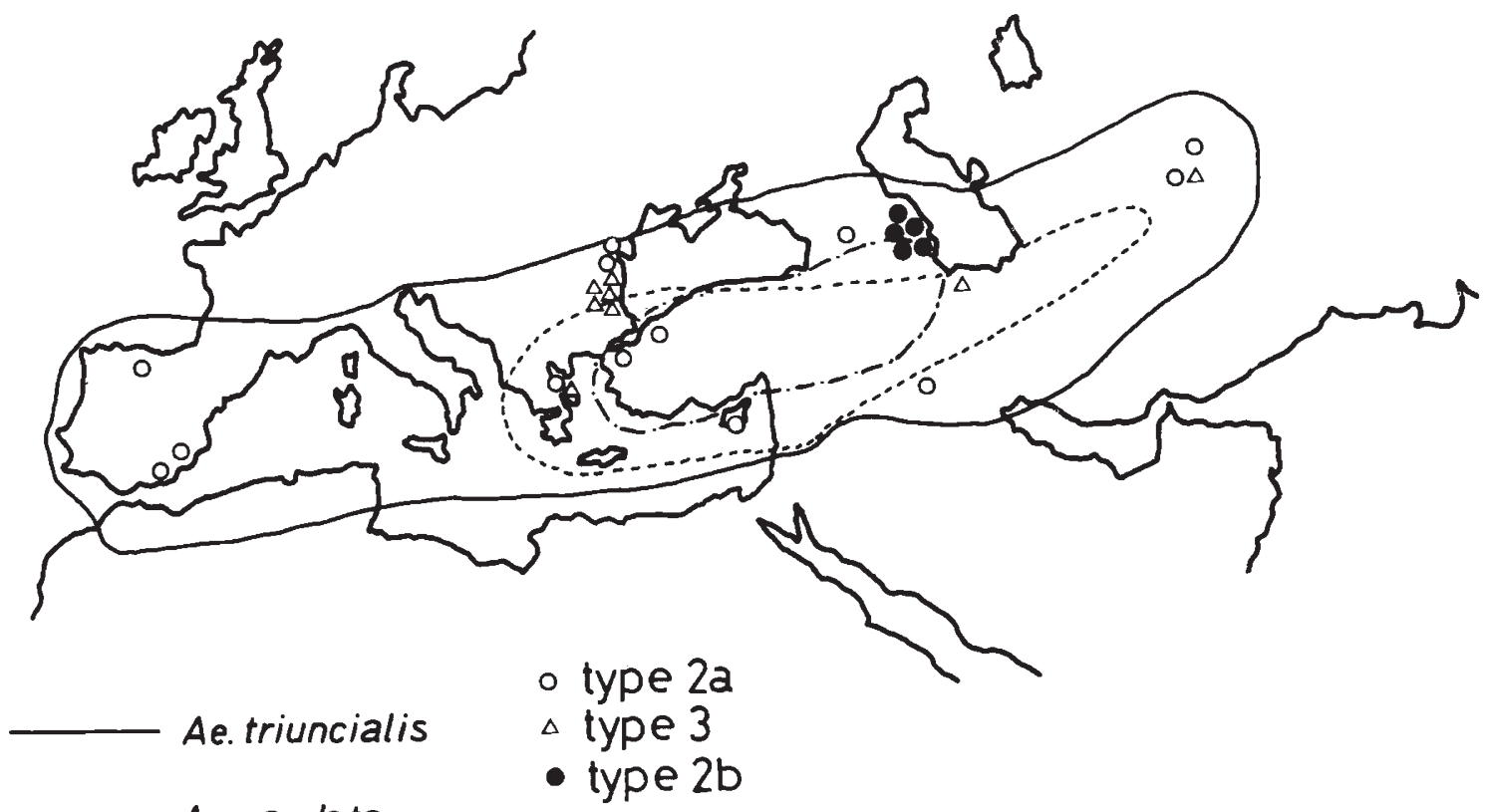

Ae. caudata

-..-.-. Ae. umbellulata

Figure 4 Geographical distribution of Ae. caudata, Ae. umbellulata and Ae.triuncialis, and collection sites of Ae. triuncialis accessions having the three chloroplast genome types.

Summarising all the results mentioned above, the following conclusions are drawn; (a) the type 2 a chloroplast genome was derived from the cross, Ae. caudata $\times$ Ae. umbellulata, while the type 3 chloroplast genome from the reciprocal cross, $A e$. umbellulata $\times A$ e. caudata, and (b) the type $2 \mathrm{~b}$ chloroplast genome originated from the type $2 \mathrm{a}$ chloroplast genome by a $0.3 \mathrm{kbp}$ insertion. This could have occurred in Ae. caudata before or in Ae. triuncialis after the origin of the latter species. A further investigation on the intraspecific variation of chloroplast genome in Ae. caudata is necessary for deciding which is the actual case.

Acknowledgements We are very grateful to Dr C. N. Law and Dr J. W. Snape, Plant Breeding Institute, Cambridge for their valuable suggestions to improve the original manuscript.

\section{REFERENCES}

Bowman, C. M., KOlleR, B., DeliUs, H. AND DYER, T. A. 1981. A physical map of wheat chloroplast DNA showing the location of the structural genes for the ribosomal RNAs and the large subunit of ribulose 1,5-bisphosphate carboxylase. Mol. Gen. Genet., 183, 93-101.

CROSTON, R. P. AND WILliams, J. T. 1981. A world survey of wheat genetic resources. IBPGR Secretariat Rome.
EIG, A. 1929. Monographish-Kritische Übersicht der Gattung Aegilops. Repert. Spec. Nov. Reg. Veget. Beich., 55, 1-228. KIHARA, H. 1940. Anwendung der Genomanalyse für die Systematik von Triticum und Aegilops. Jpn. J. Genet., 16, 309320. (in Japanese).

KIHARA, H. AND KONDO, N. 1943. Studies on amphidiploids of Aegilops caudata $\times$ Ae. umbellulata induced by colchicine. Seiken Ziho, 2, 24-42.

KIHARA, H., YAMASHITA, K. AND TANAKA, M. 1965. Morphological, physiological, genetical and cytological studies in Aegilops and Triticum collected from Pakistan, Afghanistan and Iran, pp. 1-103. In: Results Kyoto Univ. Sci. Exped. Karakoram \& Hindukush, 1955, Volume 1, Edited by $\mathrm{K}$. Yamashita, Comm. Kyoto Univ. Sci. Exped. Karakoram \& Hindukush, Kyoto.

KOLODNER, R. AND TEWARI, K. K. 1975. The molecular size and conformation of the chloroplast DNA from higher plants. Biochim. Biophys. Acta, 402, 372-390.

MUKAI, Y., MAAN, S. S., PANAYOTOV, I. AND TSUNEWAKI, K. 1978. Comparative studies of the nucleus-cytoplasm hybrids of wheat produced by three research groups. Proc. $V$ Int. Wheat Genet. Symp., 1, 282-292.

OGIHARA, Y. AND TSUNEWAKI, K. 1982. Molecular basis of the genetic diversity of the chloroplast genome and its lineage revealed by the restriction pattern of ctDNAs. Jpn. J. Genet., 57, 371-396.

OGIHARA, Y. AND TSUNEWAKI, K. 1983. The diversity of chloroplast DNA among Triticum and Aegilops species. Proc. VI Int. Wheat Genet. Symp., 407-413.

YAMASHITA, K. AND TANAKA, M. 1961. Some aspects regarding the collected materials of Triticum and Aegilops from the eastern Mediterranean countries. II. Wheat Inf. Serv. $12,24-29$. 